\title{
Efficacy of Diabetes in Pregnancy Study Group India as a Diagnostic Tool for Gestational Diabetes Mellitus in a Rural Setup in North India
}

\author{
Samar Rudra ${ }^{1}$, Ashu Yadav ${ }^{2}$
}

\begin{abstract}
Objective: To study the efficacy of Diabetes in Pregnancy Study Group India (DIPSI) as a diagnostic tool for gestational diabetes mellitus (GDM). Introduction: A simple, convenient, and patient-friendly method of diagnosing GDM by DIPSI criteria has been questioned by many workers. Hence, this study was undertaken to compare DIPSI to gold standard International Association of the Diabetes and Pregnancy Study Group (IADPSG) criteria to determine diagnostic accuracy of DIPSI.

Materials and methods: This cross-sectional study was conducted in the department of obstetrics and gynecology in a rural medical college in North India. It included 800 pregnant women with gestational age 24-28 weeks, who underwent plasma glucose (PG) evaluation 2 hours after the challenge of $75 \mathrm{~g}$ glucose load irrespective of their fasting state (DIPSI criteria for GDM). After 7 days, standard $75 \mathrm{~g}$ OGTT was done in all women irrespective of previous PG value. Blood glucose was tested by glucose oxidase peroxidase method. Accuracy of the DIPSI result was compared with OGTT using cutoffs as per standard criteria for the diagnosis of GDM.

Results: Of all 800 cases, 48 cases either did not report for the second visit in time or could not tolerate oral glucose. Of the remaining 752 cases analyzed, 620 cases found to be normal both by DIPSI and IADPSG criteria, 81 patients detected to have GDM by both criteria. In 30 patients, DIPSI detected GDM, but IADPSG criteria values were within normal limit. A total of 21 patients found to be GDM by IADPSG criteria, but DIPSI values were within normal limit. When compared with IADPSG, DIPSI found to have a sensitivity of $79.41 \%$, specificity of $95.39 \%$, positive predictive value of $72.97 \%$, negative predictive value of $96.73 \%$, and diagnostic accuracy of $93.23 \%$.

Conclusion: In conclusion, DIPSI method of screening antenatal women for GDM is found to be simple, cost-effective, easy to perform, patientfriendly, and convenient. On comparing results to gold standard IADPSG, DIPSI shows high specificity and acceptable sensitivity. A statistical analysis has shown that if a cutoff value of blood sugar is lowered to 136 from 140, the sensitivity and specificity of DIPSI criteria improve further. Keywords: Diabetes in Pregnancy Study Group India, Gestational diabetes mellitus, International Association of the Diabetes and Pregnancy Study Group, World Health Organization.

Journal of South Asian Federation of Obstetrics and Gynaecology (2019): 10.5005/jp-journals-10006-1731
\end{abstract}

\section{INTRODUCTION}

Diabetes is one of the largest global health problem of the 21st century and a lifestyle disease. Each year more and more people live with this condition, which can result in numerous complications. As economic prosperity is increasing, so is its prevalence on an increasing trend worldwide. Studies suggest that the prevalence of diabetes mellitus (DM) among women of child-bearing age group is also increasing. ${ }^{1}$

In India by World Health Organization (WHO) criteria, prevalence of gestational diabetes mellitus (GDM) is $16.55 \%{ }^{2}$ It varies from $3.8 \%$ to $21 \%$ in different parts of the country, depending on geographical locations and diagnostic methods used. ${ }^{3}$

In the Indian context, universal screening is essential in all pregnant women as the Indian women have 11-fold increased risk of developing glucose intolerance during pregnancy compared with Caucasian women. ${ }^{4}$

Increased rate of neonatal and maternal complications due to diabetes in the pregnant mothers should be considered preventable with early diagnosis during the period of gestation, so that effective treatment can be applied and adverse pregnancy outcome can be avoided.

An effective method of detecting GDM will go a long way in treating GDM and improving the perinatal morbidity, mortality, as well as maternal morbidity. In view of high prevalence rate of DM
${ }^{1}$ Department of Obstetrics and Gynaecology, DY Patil Medical College and Hospital, Pune, Maharashtra, India

${ }^{2}$ Department of Obstetrics and Gynaecology, Maharishi Markandeshwar Institute of Medical Sciences and Research, Ambala, Haryana, India

Corresponding Author: Samar Rudra, Department of Obstetrics and Gynaecology, DY Patil Medical College and Hospital, Pune, Maharashtra, India, Phone: +91 9896927925, e-mail: rudra_samar@yahoo.co.in

How to cite this article: Rudra S, Yadav A. Efficacy of Diabetes in Pregnancy Study Group India as a Diagnostic Tool for Gestational Diabetes Mellitus in a Rural Setup in North India. J South Asian Feder Obst Gynae 2019;11(6):349-352.

Source of support: Nil

Conflict of interest: None

in Indian population, the strategy of screening all the pregnant women is well accepted. The conventional two-step screening of GDM is being followed quite widely in our practice by first doing a $50 \mathrm{~g}$ oral glucose challenge test (OGCT) then oral glucose tolerance test (OGTT), if OGCT is abnormal. This strategy of screening requires the patient to visit hospital twice for detection of GDM.

The WHO first proposed criteria for GDM using a $75 \mathrm{~g}$ OGTT in the 1980s. In its technical report published in 1994, it defined GDM as DM first recognized during pregnancy, and gestational 
impaired glucose tolerance (GIGT) as impaired glucose tolerance (IGT) first recognized during pregnancy. In 1998 (published in 1999), WHO recommended new criteria. With regard to GDM, pregnant women who met the previous WHO criteria for IGT were classified as having GDM; therefore, the term "GIGT" disappeared. The WHO 1999 criteria 2-hour plasma glucose (PG) $\geq 140 \mathrm{mg} / \mathrm{dL}$ with $75 \mathrm{~g}$ oral glucose load in a fasting state gained importance in the developing countries, because it is a simple one-step procedure. ${ }^{3}$ In 2010, based on hyperglycemia and adverse pregnancy outcome study, International Association of Diabetes and Pregnancy Study Group has introduced a new set of criteria in which the threshold for making a diagnosis of GDM were lowered and recommended that GDM can be diagnosed, if any one value of fasting PG, 1-hour and 2-hour PG values meet or exceed 92, 180, and $153 \mathrm{mg} / \mathrm{dL}$, respectively, with $75 \mathrm{~g}$ oral glucose. ${ }^{5}$ Hyperglycemia and adverse pregnancy outcome study confirmed that adverse pregnancy outcome occurs with increasing maternal glucose in a continuous association even below the traditional cutoff value for diagnosis of GDM. There is a widespread acceptance of International Association of the Diabetes and Pregnancy Study Group (IADPSG) criteria including $\mathrm{WHO}^{6}$

In 2006, Diabetic Association of India recommended Diabetes in Pregnancy Study Group India (DIPSI) criteria to take 2-hour venous PG value after administrating $75 \mathrm{~g}$ of oral glucose in a non-fasting state, unlike 1999 WHO criteria in a fasting state. ${ }^{3,7}$ This is a simple single-step procedure, as generally a pregnant woman visits the antenatal clinic in a non-fasting state. Many patients come from far-flung areas, and timing and frequency of their next visit is unreliable. Considering these practical ground difficulties in our country, it is more convenient to perform the diagnostic test in a non-fasting state as recommended by the DIPSI, which is a one-step cost-effective procedure. ${ }^{8}$ However, many workers have questioned the sensitivity and specificity of DIPSI criteria in diagnosing GDM in comparison with other well-established methods. ${ }^{9-11}$ Hence, this study is carried out to compare detection rate of GDM by DIPSI over IADPSG criteria, IADPSG being most acceptable criteria internationally.

\section{Materials and Methods}

This study was carried out in a rural medical college in North India. Institutional ethical committee approval was obtained before undertaking the study. A total of 800 antenatal women were recruited for this study. Cases with known diabetic status, twin pregnancy, and those not willing to participate in the study were not considered for the study. At the time of booking, a thorough history including menstrual and obstetric history was obtained. A general physical examination, a systemic examination, and an obstetric evaluation were done. Routine investigations as per routine hospital protocol were carried out at the outset. They were called at 24-28 weeks of gestation for the study. A written consent was obtained from each patient. During the visit of the pregnant woman at 24-28 weeks of gestation, $75 \mathrm{~g}$ of glucose was given orally irrespective of their fasting state. Two-hour venous blood was taken for blood sugar estimation. The woman was asked to come after 1 week in a fasting state, and venous blood sample was drawn in a fasting state. Then, she was given $75 \mathrm{~g}$ oral glucose, and at 1 hour and 2 hours, venous blood samples were drawn. The PG was estimated in the hospital laboratory by the glucose oxidase peroxidase method. Using DIPSI criteria, GDM was diagnosed, if after $75 \mathrm{~g}$ oral glucose, 2-hour PG value equals or exceeds $140 \mathrm{mg} / \mathrm{dL}$.
Based on IADPSG criteria, GDM was diagnosed if one or more values equal or exceed thresholds of

Fasting PG of $5.1 \mathrm{mmol} / \mathrm{L}(92 \mathrm{mg} / \mathrm{dL})$.

One-hour PG level of $10.0 \mathrm{mmol} / \mathrm{dL}(180 \mathrm{mg} / \mathrm{dL})$.

Two-hour PG level of $8.5 \mathrm{mmol} / \mathrm{L}(153 \mathrm{mg} / \mathrm{dL})$.

Findings were recorded in the data sheet for further analysis.

\section{Results}

From the total 800 cases recruited, 41 women defaulted for the second visit and 7 women vomited out the glucose solution. Hence, these 48 women were excluded from the study analysis. Finally, the data of 752 women were analyzed. Our cases were from 18 years to 35 years (mean age 23.86 years) and more than $70 \%$ from 21 years to 26 years (Table 1). We have maximum cases, i.e., $73.8 \%$, with normal body mass index (BMI), overweight $12.4 \%$, obese $7.2 \%$, and underweight cases consisted of $6.6 \%$ (Table 2). Gestational DM was detected in maximum number of cases (86\%) from normal and overweight groups, which is statistically significant ( $p$ value $=0.028$ ). Gestational DM was detected in 18\% of underweight group, $12.4 \%$ of normal weight group, $22.6 \%$ of overweight group, and $22.2 \%$ of obese group (Table 3). Detection rate of GDM by DIPSI method was $14.8 \%$, and IADPSG method was $13.6 \%$ (Table 4). A cross analysis (Table 5) reveals that 81 cases (10.7\%) were found to be GDM both by IADPSG and DIPSI criteria and 620 (82.5\%) were normal by both criteria. A total of 21 cases (2.8\%) were detected GDM by IADPSG criteria, but they were normal by DIPSI criteria, whereas 30 cases (4\%) were euglycemic by IADPSG criteria but GDM by DIPSI criteria. Out of 102 cases of GDM detected by IADPSG method, 81 cases (79.4\%) was detected GDM by DIPSI but failed to detect GDM in 21

Table 1: Distribution of patients according to age

\begin{tabular}{llc}
\hline Age group in years & Distribution frequency $(n=752)$ & Percentage \\
\hline$\leq 20$ & 110 & 14.6 \\
$21-23$ & 264 & 35.1 \\
$24-26$ & 267 & 35.5 \\
$>26$ & 111 & 14.8 \\
Total & 752 & 100.0 \\
\hline
\end{tabular}

Mean age: 23.86

Table 2: Distribution of patients according to body mass index

\begin{tabular}{lcc}
\hline Group (BMI) & Distribution frequency & Percentage \\
\hline Underweight $(<18.5)$ & 50 & 6.6 \\
Normal (18.5-24.9) & 555 & 73.8 \\
Overweight (25.0-29.9) & 93 & 12.4 \\
Obese & 54 & 7.2 \\
Total & 752 & 100.0 \\
\hline
\end{tabular}

Mean BMI: 23.05

Table 3: Percentage of gestational diabetes mellitus in each body mass index group

\begin{tabular}{lcc}
\hline Group (BMI) & Distribution frequency & GDM (\%) \\
\hline Underweight $(<18.5)$ & 50 & $9(18)$ \\
Normal $(18.5-24.9)$ & 555 & $69(12.4)$ \\
Overweight $(25.0-29.9)$ & 93 & $21(22.6)$ \\
Obese $(>30)$ & 54 & $12(22.2)$ \\
Total & 752 & $111(14.8)$ \\
\hline
\end{tabular}

Chi-square $=9.6, d f=3, p=0.028$ 
Table 4: Comparison between Diabetes in Pregnancy Study Group India and International Association of the Diabetes and Pregnancy Study Group criteria for detection of gestational diabetes mellitus

\begin{tabular}{lll}
\hline GDM & DIPSI (\%) & IADPSG (\%) \\
\hline Present+ & $111(14.8)$ & $102(13.6)$ \\
Absent- & $641(85.2)$ & $650(86.4)$ \\
Total & $752(100)$ & $752(100)$ \\
\hline
\end{tabular}

Table 5: Cross-analysis of diagnostic capability of Diabetes in Pregnancy Study Group India vs International Association of the Diabetes and Pregnancy Study Group

\begin{tabular}{lllccc}
\hline & & \multicolumn{3}{c}{ IADPSG group } \\
\cline { 3 - 5 } & & & Abnormal & Normal & Total \\
\hline DIPSI group & Normal & Count & 21 & 620 & 641 \\
& & \% within IADPSG & 20.6 & 95.4 & 85.2 \\
& \multirow{3}{*}{ Abnormal } & Count & 81 & 30 & 111 \\
& & \% within IADPSG & 79.4 & 4.6 & 14.8 \\
& \multirow{2}{*}{ Total } & Count & 102 & 650 & 752 \\
& & \% within IADPSG & 100.0 & 100.0 & 100.0 \\
\hline
\end{tabular}

Table 6: Statistical data deduced International Association of the Diabetes and Pregnancy Study Group taken as gold standard

\begin{tabular}{lcl}
\hline Parameter & & $95 \% \mathrm{Cl}$ \\
\hline True-positive value & 81 & \\
False-positive value & 30 & \\
False-negative value & 21 & \\
True-negative value & 620 & \\
Sensitivity & $79.41 \%$ & $70.57-86.12$ \\
Specificity & $95.4 \%$ & $93.5-96.75$ \\
Positive predictive value & $72.97 \%$ & $59.0-83.5$ \\
Negative predictive value & $96.73 \%$ & $93.8-98.2$ \\
Diagnostic accuracy & $93.23 \%$ & $91.2-94.81$ \\
\hline
\end{tabular}

cases (20.6\%). Out of the 650 of normal cases by IADPSG criteria, 620 cases (95.4\%) were normal by DIPSI criteria and 30 cases (4.6\%) were abnormal. IADPSG criteria taken as gold standard, we found truepositive 81 cases, false-positive 30 cases, false-negative 21 cases, and true-negative 620 cases when DIPSI criteria was employed on the same subjects. When compared with IADPSG, DIPSI has a sensitivity of $79.41 \%$, specificity of $95.39 \%$, positive predictive value of $72.97 \%$, negative predictive value of $96.73 \%$, and diagnostic accuracy of $93.23 \%$ (Table 6).

When results were plotted in a receiver operating characteristic curve, area under curve is 0.963 [95\% confidence interval $(\mathrm{Cl})$, $0.963-0.985$ ], which is highly significant ( $p$ value $<0.001$ ) (Fig. 1$)$.

Dichomatizing the results as "negative" or "positive" using different cutoff points applying the WinPepi analysis, Youden's index found to be highest at a cutoff point of DIPSI at 136, where sensitivity will come to $94.1 \%$ and specificity to $91.7 \%$.

\section{Discussion}

Universal screening of all pregnant women for GDM in India is a well-accepted strategy. However, controversy arises on choosing the method of screening. International Association of the Diabetes and Pregnancy Study Group has a widespread acceptance including WHO. ${ }^{6}$

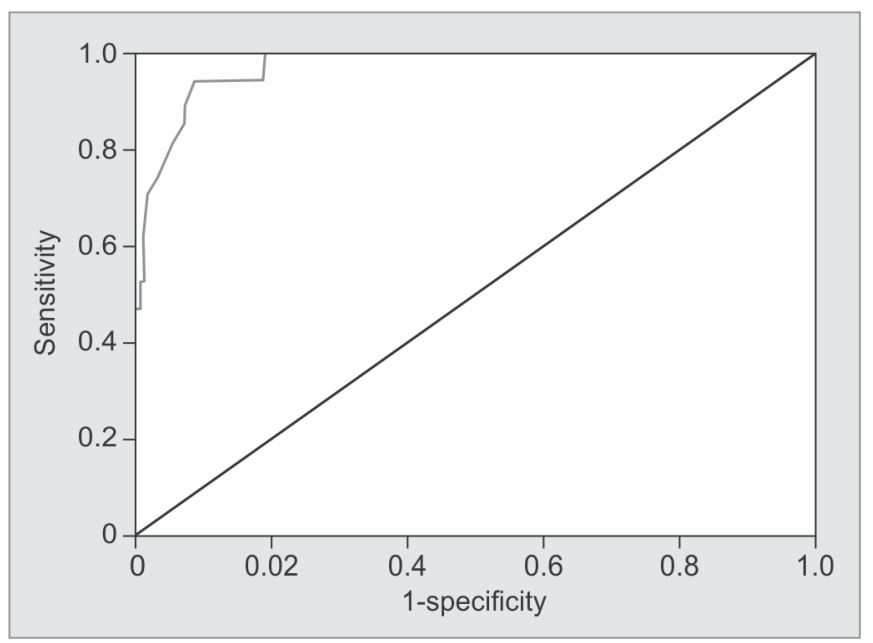

Fig. 1: Receiver operating characteristic curve. Area under curve is 0.963 (95\% confidence interval $0.963-0.985)$ which is highly significant ( $p$ value $<0.001)$

We conducted this study to diagnose GDM both by DIPSI and IADPSG criteria and did a comparative analysis of DIPSI results with that of IADPSG. We found DIPSI as a diagnostic tool to detect GDM has a sensitivity of $71.4 \%$ and specificity of $95.4 \%$ when compared with IADPSG criteria. Our positive predictive value is $73 \%$ and negative predictive value $97 \%$ and diagnostic accuracy $93 \%$. A similar study conducted on 936 pregnant women by Tripathi et al. ${ }^{9}$ had sensitivity value of $74.1 \%$ and specificity value of $96.9 \%$ though they did not recommend DIPSI, as it missed a few and overdiagnosed a few GDM when compared with both WHO and IADPSG criteria. It is pertinent to mention here that, in their study, only 35 cases were picked up as GDM by all three criteria from 64, 63, and 73 cases of GDM diagnosed individually by IADPSG, WHO, and DIPSI criteria, respectively. When IADPSG and WHO values were compared, only 38 cases were GDM by both IADPSG and WHO criteria from 64 and 63 cases, respectively. Needless to mention here that every criteria bound to have some false-positive and -negative results. 
In another study done by Mohan et al., ${ }^{10}$ DIPSI has a poor sensitivity compared with both the WHO 1999 criteria (27.7\%) and the IADPSG criteria (22.6\%). It was found to miss $72.3 \%$ of women with GDM diagnosed by the WHO 1999 criteria and $77.4 \%$ of women with GDM diagnosed by the IADPSG criteria. Vij et al. ${ }^{11}$ in a similar study like us compared DIPSI with IADPSG and did not find DIPSI a satisfactory method though they diagnosed $74.34 \%$ of cases by DIPSI. Moreover, their sample size was small.

However, many other studies ${ }^{8,12,13}$ found DIPSI as useful criteria to detect GDM especially in a country like India. Nallaperumal et al. ${ }^{8}$ argued in their study that in IADPSG criteria, the low value of fasting may overdiagnose and high value of 2 hours may miss a few cases of GDM especially in Indian scenario. They have found 2-hour OGTT value is more sensitive than fasting blood sugar (FBS) value in nonpregnant Indian women in one of their studies. Polur et al. ${ }^{12}$ found, in Indian context, DIPSI is a useful method when compared with WHO criteria. They could pick up $98 \%$ of GDM. Magon et al. ${ }^{13}$ had also recommended the DIPSI test for universal use in India. Sharma et al. ${ }^{14}$ in 2019 found similar finding like us in their study conducted in North India.

On further analysis of our data revealed that sensitivity and specificity are highest when cutoff point of DIPSI is taken at 136, which will improve the accuracy of DIPSI as a criterion to diagnose GDM.

\section{Conclusion}

In conclusion, DIPSI method of screening antenatal women for GDM is found to be simple, easy to perform, convenient, and well accepted by the patient. When results were compared with gold standard IADPSG, DIPSI shows high specificity and acceptable sensitivity. Further analysis of our data revealed that by bringing down the cutoff value of DIPSI to 136 will further improve the accuracy of DIPSI as a method of diagnosis of GDM in our pregnant women. A large multicentric study is necessary to substantiate our observation.

\section{References}

1. Tutino GE, Tam WH, Yang $X$, et al. Diabetes and pregnancy: perspectives from Asia. Diabet Med 2014;31(3):302-318. DOI: 10.1111/ dme.12396.
2. Rajput R, Yadav Y, Nanda S, et al. Prevalence of gestational diabetes mellitus \& associated risk factors at a tertiary care hospital in Haryana. Indian J Med Res 2016;137(4):728-733.

3. Seshiah V, Das AK, Balaji V, et al. Diabetes in pregnancy study group. gestational diabetes mellitus - guidelines. J Assoc Physicians India 2006;54:622-628.

4. Neelakandan RP. Early universal screening for gestational diabetes mellitus. J Clin Diagnostic Res 2014;8(4):12-14. DOI: 10.7860/ JCDR/2014/8199.4264.

5. Joshi SR, Gupta S, Gupte S, et al. Diagnosis and management of gestational diabetes mellitus: indian. Assoc Physician India [Internet] 2013. 202-204. Available from: http://www.apiindia.org/.

6. Polur $\mathrm{H}$, Rrora R, Bandela PV. A minireview on diagnostic criteria of gestational diabetes mellitus (GDM). J Pharm Sci Res 2015;7(8): 538-541.

7. Reddi Rani P, Begum J. Screening and diagnosis of gestational diabetes mellitus, where do we stand. J Clin Diagnostic Res 2016;10(4):QE01-QE04.

8. Nallaperumal S, Bhavadharini B. Comparison of the world health organization and the international association of diabetes and pregnancy study groups criteria in diagnosing gestational diabetes mellitus in South Indians. Indian J Endocr Metab 2013;17(5):906-909. DOI: $10.4103 / 2230-8210.117241$.

9. Tripathi R, Verma D, Gupta VK, et al. Evaluation of $75 \mathrm{~g}$ glucose load in non-fasting state [diabetes in pregnancy study group of India (DIPSI) criteria] as a diagnostic test for gestational diabetes mellitus. Indian J Med Res 2017;145(2):209-214.

10. Mohan V, Mahalakshmi MM, Bhavadharini B, et al. Comparison of screening for gestational diabetes mellitus by oral glucose tolerance tests done in the non-fasting (random) and fasting states. Acta Diabetol 2014;51(6):1007-1013. DOI: 10.1007/s00592-014-0660-5.

11. Vij P, Jha S, Gupta SK, et al. Comparison of DIPSI and IADPSG criteria for diagnosis of GDM: a study in a North Indian tertiary care center. Int J Diabetes Dev Ctries 2015;35(3):1-2. DOI: 10.1007/s13410-014-0244-5.

12. Polur $\mathrm{H}$, Prasad KD, Bandela PV, et al. Diabetes in pregnancy study group in India (DIPSI) - a novel criterion to diagnose GDM. Int J Biochem Res Rev 2016;10(1):1-6. DOI: 10.9734/JJBCRR/2016/22624.

13. Magon N, Chauhan M, Diagnosing GDM. Role of simple, cost effective, and sensitive DIPSI test. J Obstet Gynaecol India 2014;64(4):299-300. DOI: 10.1007/s13224-014-0594-4.

14. Sharma N, Pundir S, Dinani B. Can single plasma glucose value 2 hours after $75 \mathrm{~g}$ glucose (DIPSI criteria) replace the gold standard OGTT for the diagnosis of gestational diabetes mellitus? Int J Reprod Contracept Obstet Gynecol 2019;8(3):1041-1046. DOI: 10.18203/23201770.ijrcog20190877. 